\section{La fragmentación de los sistemas nacionales de salud}

\author{
Edgar Barillas ${ }^{1}$
}

1 Consultor de la Organización Panamericana de la Salud en Guatemala, Área de Políticas y Sistemas de Salud.

2 Dentro de los acuerdos de paz suscritos en Guatemala, en el de "Reasentamiento de las Poblaciones Desarraigadas por el Conflicto Armado" se establece la necesidad de desarrollar modelos locales y autóctonos de servicios de salud.
En el mundo de hoy se cuestionan muchos de los pilares que sustentan la organización de la sociedad. No es raro encontrar ejemplos de países donde se han legitimado las diferencias y se ha puesto en duda la viabilidad del Estado-nación, dando lugar a nacionalismos que parecían haber sido sepultados bajo el manto de los siglos. Un factor agravante en tales casos es que esta renovada conciencia de identidad étnica y cultural no necesariamente guarda relación con las divisiones político-administrativas de los países, como demuestra el claro ejemplo del conflicto armado y la posterior división de la antigua Yugoslavia.

Sin duda la ruptura social es un fenómeno cada vez más frecuente que se fundamenta no solo en el resurgimiento de nacionalismos de raíz cultural y étnica, sino también en la estratificación que produce la desigual distribución de la riqueza y en los procesos de descentralización conducidos por el propio Estado (Alvarado F, Comunicación personal, enero de 1996). Más aún, dado que estos factores desencadenantes no coinciden entre sí en su origen y propósito, el resultado de su confluencia será la escisión del tejido social en grupos que comparten características muy específicas.

En países multiétnicos como Guatemala, probablemente el más influyente de los factores causales del proceso de fragmentación social se origina en la reivindicación de los valores culturales propios de los distintos grupos de población. En el área de los servicios de salud, esta reafirmación cultural puede llevar a la creación de modelos autóctonos en los cuales tendrán mayor peso los valores y preferencias sociales de cada comunidad. ${ }^{2} \mathrm{Si}$ esto ocurre, podría darse el caso de que en un país convivan numerosos sistemas de salud entre los cuales existe poca relación estructural y funcional. Las demandas de desarrollo local de las distintas comunidades se expresarán en sistemas de salud con una autonomía y una identidad tan particulares, que harán difícil vincularlos a un sistema nacional integrado.

Por otra parte, la nueva ola de políticas de liberalización económica también puede provocar una fragmentación social al acentuar las diferencias en el ingreso, el consumo y la posesión de bienes de capital. Esta recomposición social - que de hecho tiende al individualismo extremo- configura modelos de atención de salud que difieren de acuerdo a la capacidad de pago del usuario. Parece poco probable, sin embargo, que aun en el marco de 
la aplicación de las políticas neoliberales más extremas, el sistema evolucione hacia el completo individualismo en la compra de servicios de salud. En otras palabras, es prácticamente imposible que en el futuro todo el gasto de salud sea financiado directamente del bolsillo del usuario, sobre todo si se tiene en cuenta que el costo de los servicios de salud usualmente supera las capacidades económicas individuales o familiares. Las características del proceso salud-enfermedad hacen que los servicios de salud por lo general requieran ser financiados por un sistema de seguro.

No obstante, en un escenario de fragmentación social, los seguros de salud no pueden alcanzar una escala nacional. Lo más probable es que se conformen círculos de solidaridad determinados por la capacidad de pago de los consumidores. Entonces, progresivamente se establecerán seguros de salud empresariales, gerenciales, obreros, campesinos, etc., cada uno diferenciado por el monto del aporte de sus miembros, es decir, por la cantidad y calidad de las prestaciones que ofrecen. De este modo la solidaridad que se genera dentro de un seguro campesino, por ejemplo, es socialmente más deseable que afrontar el costo de los servicios de salud individualmente o con recursos familiares. ${ }^{3}$

La meta de eficiencia y equidad que se persigue en el campo de los servicios de salud, sin embargo, no se limita a la solidaridad que puede generar un club cuyos socios comparten la misma posición económica, sino que se extiende a la sociedad en su conjunto. Los esfuerzos de los países deben encaminarse a la creación de un sistema de salud donde el sano subsidia al enfermo, pero sobre todo donde el rico subsidia al pobre.

Los esquemas de seguro fragmentados contribuyen, además, a aumentar la falta de equidad y el gasto del sistema de salud, entre otras por las razones que se detallan a continuación.

- La reducción de la distribución del riesgo poblacional mediante la formación de grupos más restringidos de asegurados.

- El menor grado de solidaridad del sistema, dado que en las comunidades de ingresos más elevados la cobertura será mayor que en las más pobres.

- El aumento del costo de los servicios, al desarrollarse múltiples unidades administrativas periféricas. Los costos administrativos pueden ser aún mayores si las burocracias centrales no se desmantelan adecuadamente.

\footnotetext{
3 En Guatemala recientemente se han creado seguros de campesinos en los municipios de Ixcán y Barillas. Según los cálculos actuariales, el costo anual de cubrir una canasta mínima familiar de servicios esenciales se ha estimado en US\$ 17,00 .
}

- El aumento de los costos derivado de la compra independiente de servicios a proveedores privados. En estas circunstancias los servicios tendrán, además de mayores gastos administrativos, el costo agregado de promoción y mercadeo. ${ }^{4} \mathrm{La}$ experiencia de varios países desarrollados demuestra, además, que mientras más centralizada es la administración de los servicios de salud, más fácil resulta controlar los costos, y que cuanto más reducido es el número de fuentes de financiamiento, menores son los gastos administrativos.

\section{LA SITUACIÓN EN AMÉRICA LATINA}

Varios países latinoamericanos están llevando a cabo un acelerado proceso de descentralización administrativa y financiera de los sistemas de salud. El objetivo es hacer que los recursos lleguen más cerca del usuario, generando así una mayor aproximación entre la demanda de la población y la respuesta del Estado. También se argumenta que en el mismo proceso se deben fortalecer la capacidad de conducción y regulación del nivel central, la participación comunitaria y el desarrollo democrático.

En la práctica, la descentralización no parece haberse acompañado del fortalecimiento necesario del nivel central, en parte porque las herramientas para impulsar el proceso de descentralización están mejor desarrolladas que aquellas que deben, paralelamente, fortalecer la función rectora del Estado. De hecho, se han cedido paulatinamente recursos de poder que inhabilitan al nivel central para ejercer sus funciones tradicionales. No hay que olvidar que el control de los recursos financieros no es solamente un mecanismo para la redistribución presupuestaria con criterios de equidad, sino también un medio coercitivo para la observancia de la gestión pública. La descentralización presupuestaria hace que el nivel central pierda su capacidad de intervención en la periferia. Asimismo, junto con la disminución de los fondos se deteriora la capacidad técnica del nivel central, perdiéndose otro recurso de poder que antes poseía. Finalmente, la influencia del sector de la salud sobre los altos niveles políticos también se verá mermada en beneficio de los entes descentralizados que, mejor dotados de recursos y capacidad técnica, resultan interlocutores con mayor poder de decisión e influencia.

\footnotetext{
4 En un estudio efectuado en varios países desarrollados se concluye que la mayor participación del sector privado en el financiamiento de un sistema de salud disminuye la eficiencia y la capacidad de controlar los costos. (Economic Council of Canada. Regulatory mechanisms in the health care systems of Canada and other industrialized countries: description and assessment. ECC; 1991.)
} 


\section{LOS PROBLEMAS DE CONDUCCIÓN DEL ESTADO}

Las propuestas de reforma del sector de la salud hasta ahora diseñadas en la mayor parte de los países dependen de la capacidad rectora de un ministerio de salud que propone modelos alternativos de atención, elabora canastas básicas, enfoca la atención en grupos de riesgo, otorga la prestación de ciertos servicios a agentes privados y descentraliza la administración de los servicios. Los procesos de reforma concebidos con tales premisas no han sido muy exitosos, aun en aquellos casos en que se ha contado con un sólido respaldo técnico de las propuestas. Es probable que la causa radique en la referida carencia de recursos de poder de la entidad rectora - los ministerios de salud - para impulsar la transformación requerida en los sistemas. Paradójicamente, en una coyuntura histórica que torna imprescindible introducir cambios en el sistema de salud, el Estado se encuentra más debilitado que nunca. La avalancha neoliberal ha deteriorado su solvencia financiera y su credibilidad, los mejores cuadros técnicos han migrado hacia el sector privado y la influencia del sector social en el ámbito político ha perdido fuerza.

En estas circunstancias, la dirección de los procesos de reforma queda transitoriamente en manos de quienes efectivamente poseen los mencionados recursos de poder. Los organismos internacionales son ahora actores influyentes en la reforma sectorial al poner a disposición de los países recursos financieros y técnicos para impulsarla.

Los programas para el sector de la salud propuestos por las instituciones internacionales promueven la canalización de los recursos públicos a los grupos de población más pobres y a las intervenciones más efectivas en función de los costos. Dado que la ineficiencia es un problema sentido para un sector que tiene que responder con menos recursos a las demandas crecientes de la población, tales programas son en general bienvenidos por los círculos técnicos y políticos de los países beneficiarios. No hay, al parecer, ninguna razón para que los gobiernos rechacen propuestas técnicas sólidamente diseñadas y congruentes con los intereses nacionales. El problema se plantea cuando un ministerio de salud debilitado tiene que hacerse cargo de una empresa que no pudo encarar incluso en momentos de mayor fortaleza. A primera vista la solución podría ser el robustecimiento de los ministerios de salud antes de emprender, bajo su liderazgo, los cambios sectoriales propuestos. La pregunta es si el Estado - en este caso el ministerio de salud- puede fortalecerse dadas las actuales tendencias hacia el regionalismo subnacional, la autodeterminación, el rechazo a las respuestas preelaboradas, las decisiones individuales y la libertad de elección.

¿Cuál sería el futuro de los servicios de salud si estas tendencias fueran aceptadas como un hecho inexorable y el Estado se replegara para dar paso a formas autóctonas de organización de los servicios de salud? ¿Tendríamos un sistema de servicios más eficiente y equitativo? ¿Qué pasaría si las autoridades de salud fueran elegidas localmente, para administrar conforme a disposiciones locales los recursos financieros generados en ese nivel? Tal vez así el sistema de salud respondería mejor a las necesidades locales, pero ¿sería más solidario y eficiente?

\section{OTRAS CONSIDERACIONES SOBRE EL PAPEL DEL ESTADO}

Independientemente del poder con que puedan influir los factores desencadenantes descritos, la descentralización y la autonomía política y financiera - llevadas a extremos y sin una conducción idónea- causan una fragmentación del sistema de salud, que anula cualquier expectativa de conseguir un modelo eficiente y solidario. Un sistema que aspira a la equidad debe promover, como se ha mencionado, un subsidio del rico hacia el pobre y del sano hacia el enfermo. Con la fragmentación del sistema de salud los grupos de población de mayores ingresos abandonarían los seguros públicos para trasladarse a esquemas privados en los cuales recibirían una atención más acorde con su capacidad de aporte.

Al fragmentarse los sistemas se pierden además las economías de escala derivadas de la adquisición de insumos en grandes cantidades, particularmente medicamentos y materiales medicoquirúrgicos. Por otra parte, la administración del sistema de salud también está sujeta a las mismas economías de escala, debido a que cuanto más grandes son los grupos de población menores son los gastos administrativos proporcionales.

Otra consideración importante se refiere a las políticas públicas de alcance nacional, cuya ejecución se dificultaría en una situación de fragmentación progresiva. Si se otorga a las comunidades el derecho a configurar el sistema de salud que mejor responda a sus expectativas, declina la ingerencia del nivel central en las decisiones de política. Este hecho podría tener repercusiones desfavorables en programas que deben realizarse a escala nacional para lograr el máximo impacto, como el control de vectores, la inmunización y el saneamiento ambiental.

Si a las consideraciones del presente análisis se suman los problemas de la prestación privada de 
servicios de salud, los más elementales principios de justicia social y la experiencia de los sistemas de salud de otros países, no resulta difícil comprender el beneficio de adoptar sistemas de salud unificados administrativamente $\mathrm{y}$ financiados por el sector público. El reto que se plantea entonces es lograr que haya compatibilidad entre una poderosa y legítima tendencia hacia la fragmentación social con la eficiencia y equidad que buscan los sistemas de salud. La pregunta no es solo qué debe sino qué puede hacer el Estado en estas circunstancias. En primer lugar el Estado, desde el nivel central, debería asegurar el control directo de una buena proporción del presupuesto nacional con dos propósitos fundamentales: contar con un fondo que permita la redistribución presupuestaria sobre la base de criterios de postergación y disponer de recursos que sirvan para activar la política pública en el plano nacional. La transferencia de fondos centrales a los gobiernos locales, sujeta al cumplimiento de ciertas metas de salud y a la observancia de principios elementales de eficiencia y equidad, puede favorecer la coherencia del sistema de salud, aun en escenarios de relativa autonomía del nivel local.

Para poner en marcha una estrategia como esta, el Estado debe desarrollar centralmente la capacidad técnica necesaria para evaluar la eficiencia de las intervenciones que se ensayan localmente. Un análisis comparado del funcionamiento de los servicios en las distintas comunidades permitirá, en el nivel central, orientar la asignación de las partidas presupuestarias, y en el plano local, consolidar o modificar las acciones adoptadas. Por lo tanto, el nivel central debe tener a su cargo la formulación de la política nacional de salud y la generación de información técnica que permita su seguimiento y evaluación, facilitando asimismo que localmente se efectúe una programación más afín con la normativa nacional. También compete al Estado establecer un amplio sistema de información al público que facilite el control social de la administración de los servicios de salud y la adopción de las decisiones individuales más ventajosas respecto al cuidado de la salud y la demanda oportuna de servicios asistenciales.

Las comunidades y grupos organizados deben, por su parte, mantener la autonomía que han logrado. El éxito de un sistema de salud organizado y financiado localmente depende no solo de la identidad cultural, religiosa e idiomática, sino también de la eficiencia y solidaridad que el sistema demuestre. Un elemento fundamental para el mantenimiento de una relativa autonomía local es la generación de recursos financieros adicionales en la misma comunidad. Los sistemas locales de salud tienen que mostrar un alto grado de solidaridad interna, que puede a su vez ser uno de los principios nacionales para el mantenimiento de un sistema coherente y articulado. Por último, en el nivel local hay que asegurar una amplia participación de la población en la toma de decisiones y en la fiscalización de los servicios, ya que tal participación ha sido el argumento fundamental a favor de un modelo local y autónomo de servicios de salud.

\section{SYNOPSIS}

\section{The fragmentation of national health systems}

The nationalistic tendencies observed in the world today, rooted in ethnic and ancestral cultural values that do not necessarily coincide with the physical boundaries of countries, have an enormous influence on societal organization, the distribution of wealth, and the processes of decentralization being carried out by governments. At the same time, the economic liberalization policies which are in fashion accentuate social divisions and favor models of health care that are fragmented according to the economic means of the user. This document explores the consequences of these trends for equity in the health and medical insurance systems of Latin America, as well as the role of the State and the private sector in the provision of services. 